\title{
Devant la loi
}

\section{Gérard Timsit et Pierre Livet}

\section{OpenEdition \\ Journals}

Édition électronique

URL : http://journals.openedition.org/ress/361

DOI : 10.4000/ress.361

ISSN : 1663-4446

\section{Éditeur}

Librairie Droz

\section{Édition imprimée}

Date de publication : 1 janvier 2006

Pagination : 7-12

ISBN : 2-600-00959-0

ISSN : 0048-8046

Référence électronique

Gérard Timsit et Pierre Livet, « Devant la loi », Revue européenne des sciences sociales [En ligne], XLIV-133 | 2006, mis en ligne le 12 novembre 2009, consulté le 05 mai 2019. URL : http:// journals.openedition.org/ress/361 ; DOI : 10.4000/ress.361 


\section{Gérard TIMSIT \\ Pierre LIVET}

\section{DEVANT LA LOI}

En retenant «Devant la loi » comme thème fédérateur de notre réflexion pour Arolla 2005, il semble que notre Groupe ait souhaité manifester son souci qu'une double synthèse puisse être faite à cette occasion:

- d'abord, entre les thèmes évoqués de divers côtés à la fin de la réunion d'Arolla 2003: la résistance, l'inéluctable, la souffrance, l'inaccessibilité, l'instrumentalisation, l'insignifiance, le proche et le lointain, etc..

- ensuite, peut-être moins explicitement, mais encore assez nettement, semblet-il, une synthèse en forme de rappel ou de bilan de thèmes évoqués dans les réunions antérieures du Groupe: la loi, la métaphore, la preuve, la peur de l'impensable, etc...

Selon les habitudes de notre Groupe, cette réflexion doit se faire par le repérage de l'incidence des différents thèmes à la fois dans nos disciplines et dans les phénomènes contemporains tels qu'ils se signalent aux disciplines dont nous relevons.

Présentant le rapport à la loi comme un rapport à ce qui est à la fois incompréhensible et impénétrable, mais qui pourtant, si nous l'avions compris, était fait pour nous, la nouvelle de Kafka donne à voir l'absurde des situations où nous nous trouvons placés, qui tient au refus ou à la dépossession du sens dont nous sommes victimes lorsque ce que nous ne comprenons pas se révèle pourtant déterminer notre destin - situations qui engendrent un malaise ou un mal-être, parce qu'elles sont duales. D'une part elles se proposent comme mettant en jeu ce qui règle nos interactions et donne à nos coordinations leurs repères, à nos litiges leurs aboutissements, d'autre part elles sont vécues comme ce qui méconnaît nos aspirations et dénie nos tentatives de donner sens et valeurs à nos interactions. Il ne s'agit pas là de l'acceptation nécessaire, à la manière stoïcienne, de circonstances auxquelles nous ne pouvons rien et qui s'opposent à nos tentatives de trouver du sens à nos actions. Nous resterions alors une source de sens - la seule - même si elle est faible et incertaine. En vérité, ce sont les institutions mêmes sans lesquelles nous ne pouvons tout simplement pas donner de repères à nos interactions qui sont à la fois le lieu obligé de notre recherche de valeurs, et les producteurs d'un déni de cette recherche.

«Devant la loi » n'est cependant pas - le titre de la nouvelle le montre bien l'analyse de l'état ou de la structure de la loi en soi mais de son fonctionnement et de ses dysfonctionnements: de la relation créée - et qui manque de fonctionner - entre la Règle et celui qui doit la respecter. Sa signification est incompréhensible 
à l'homme de la campagne. Et pourtant, la règle, qui est générale, «ne doit-elle pas être accessible à tous et toujours »? Elle ne l'est pas. Elle le serait devenue, pourtant - si l'homme de la campagne avait compris qu'une entrée, qui n'était faite que pour lui, existait qui lui eût permis d'y accéder. Description d'une relation où s'affrontent, se combattent - et échouent à s'associer - la généralité de la règle et la singularité de l'accès qui en eût permis la compréhension. Description d'une relation de signification - ou, plus exactement, des conditions dans lesquelles échoue à se transporter une signification - un message - d'un individu, ou d'un site, à un autre. C'est tout le problème de l'interprétation de la loi - qui n'est pas le seul que pose en l'occurrence la question du transfert de sens: ce que permet de percevoir la confrontation de cette nouvelle de Kafka à une autre de ses nouvelles, tout aussi courte et aussi suggestive: «Un message impérial»,

Dans cette deuxième nouvelle, il s'agit d'un message, adressé par l'Empereur - «à toi, le plus lamentable de ses sujets », dit le récit. L'Empereur est sur son lit de mort, prêt à s'éteindre, à disparaître en même temps que s'apprête à s'éloigner son messager, porteur du message qu'il lui a remis et qu'il lui a fait répéter à son oreille, avant de partir, pour s'assurer de son exactitude. Le messager se met en route, à travers la foule - «mais la foule est si grande...» Il traverse cours et palais, d'autres cours, des palais, et des cours et d'autres encore, et ainsi pendant des millénaires, et s'il parvenait à l'ultime portail - «mais jamais, non jamais il n'y parviendra »-, il y aurait encore devant lui «la ville impériale tout encombrée de sa propre lie». Or, là, «personne ne pénètre avec le message d'un mort». Nouvelle horrible histoire d'inaccessibilité - étrangement symétrique et inverse de la précédente. Dans les deux cas, l'épuisement du sens se traduit dans la mort - de l'homme de la campagne, qui ne peut entrer dans la loi ; - de l'Empereur, dont le messager ne pourra faire parvenir le message à son destinataire, si misérable soitil. Mais, cette fois, le sens est porté non plus par la loi mais par le messager - deux institutions, certes, mais dont l'une est anonyme tandis que l'autre s'incarne dans un individu. Au delà du problème de l'interprétation, la question de la représentation: deux manières pour les institutions de fonctionner - ou non. Nous pensions que les institutions étaient faites pour garantir nos libertés dans leurs co-existences aussi ouvertes que possible. Montesquieu et Roussseau nous l'avaient assuré $^{1}$. Nous nous apercevons que ces institutions sont très efficaces pour assurer des coordinations, mais qu'en revanche les effets systémiques de ces coordinations rendent très difficiles la simple exploration d'autres possibilités d'interactions. Notre ingénierie sociale a construit des systèmes qui gèrent non plus seulement des choses, mais des règles et des instances de signification. Or ces systèmes fonctionnent de manière aussi aveugle que des choses. Nous nous sentons donc dépossédés de nos tentatives d'apporter ou reconnaître des significations, puisque ce qui sanctionne leur acceptation sociale, c'est leur transmission par les systèmes, dont les effets collectifs peuvent ne rien avoir à faire avec l'orientation de ces tentatives.

Dans les sciences sociales et les domaines qu'elles gèrent ou dont elles prétendent assurer la connaissance, se manifeste le plus clairement ce malaise. Et c'est

Du moins nous a-t-on dit qu'ils l'avaient assuré. Il n'est pas certain qu'ils n'aient pas quelque peu nuancé leurs assurances... 
ce malaise qu'il nous appartient de tenter de cerner en repérant les paradigmes autour desquels se construisent la relation de signification et les dysfonctions auxquelles elle donne lieu - des paradigmes qui peuvent être analysés à deux niveaux: celui de la structure de la relation, et celui du rôle dévolu aux analyses de ce type de relations.

1. Le récit - parabole (?), métaphore (?) - de Kafka² attire l'attention sur trois couples d'oppositions autour desquels se structure la relation de signification:

- la structure de la relation elle-même, considérée dans son ensemble comme relation du général au particulier. Une première opposition se fait jour - entre concept et contexte: les concepts nécessairement utilisés par la loi générale et impersonnelle pour être ce qu'elle est et atteindre à la généralité dont elle est porteuse, qui lui permet de s'adresser à tous; et le contexte nécessaire à son application singulière aux individus qui en sont les destinataires et ne peuvent recevoir application de la loi - ne peuvent y accéder - que s'ils passent par l'entrée qui, dans la loi, leur est à eux seuls réservée.

Cette première opposition permet d'esquisser l'analyse d'un double dysfonctionnement de la loi. Celui de la régression vers une sur-abstraction: une totale «dématérialisation» de la loi - ce qui peut se décliner sur deux modes: le mode majeur de la loi comme «simple forme», alors « réduite au degré zéro de sa signification $»^{3}$ et sans contenu déterminable: une loi «qui est en vigueur mais ne signifie pas » et prépare les voies des sociétés totalitaires (Agamben); ou bien sur le mode mineur, une loi qui énonce des principes tellement généraux qu'ils ne peuvent en acquérir la moindre utilité sociale (ce qui pourrait être le cas, dans certaines formulations juridiques, du principe de précaution). Le deuxième dysfonctionnement pourrait, à l'inverse, s'analyser en une fuite en avant - vers une sur-figuration, une sur-contextualisation, dont une des formes privilégiée et paradoxale (parmi bien d'autres, faciles à discerner) serait l'empilement de dispositions normatives destinées à régler les moindres détails de la vie collective - ce que l'on appelle habituellement, chez les juristes, l'inflation normative.

- la structure de la relation considérée maintenant dans sa destination - le particulier. Deuxième opposition, entre le particulier considéré comme exemple, application du général, et par conséquent inclusion (subsomption) sous le général, et l'exception - qui est une «espèce de l'exclusion» (Agamben, I. 25), puisque située par hypothèse hors de la norme, sans que pour autant elle nie tout rapport avec elle: «au contraire, celle-ci se maintient en relation avec elle sous la forme de la suspension» (ibid.).

Naissent toute une série de problèmes:

«Devant la loi» a fait l'objet, à notre connaissance, de trois commentaires récents: J. Derrida, Préjugés, devant la loi, in: J. Derrida et al. La faculté de juger, Ed. de Minuit, 1985; M. Cacciari, Icones de la loi, Paris 1990; G. Agamben, Homo sacer, Le pouvoir souverain et la vie nue, Seuil 1995.

G. Agamben, précité, p. 61 
- de l'uniformité de la loi et de la diversité de ses applications, respectueuses, ou non (si ce sont des exceptions), de sa généralité;

- de la mesure dans laquelle les applications respectueuses de la généralité de la loi la confirment: question du continuum des applications - de l'exemple (ce que Kelsen ne connaît et ne conceptualise que sous les espèces de la concrétisation du droit) à la dérogation sous les diverses formes qu'elle revêt ( de la «discrimination justifiée» à la française à l'affirmative action ou «discrimination positive à l'américaine, etc...);

- de la localisation de l'exception dans l'ordre juridique, dont elle peut être analysée soit, de façon classique, comme négation, soit à la manière de Carl Schmitt, comme fondation...

- la structure de la relation considérée dans sa référence - la généralité. L'opposition se fait désormais entre les modes d' «accès » à la généralité - dans le vocabulaire kantien, par «réflexion» ou «réfléchissement» (?). Le problème se pose alors de la nature de l' «accès » et de son rapport à la justice: «si l'acte consiste simplement à appliquer une règle, à dérouler un programme ou effectuer un calcul, on le dira peut-être légal, conforme au droit et peut-être par métaphore, juste, mais on aurait tort de dire que la décision a été juste (...). Pour qu'une décision soit juste et responsable, il faut que, dans son moment propre, s'il y en a un, elle soit à la fois réglée et sans règle, conservatrice de la loi et assez destructrice et suspensive de la loi pour devoir, à chaque cas, la réinventer, la rejustifier, la réinventer au moins dans la réaffirmation et la confirmation nouvelle et libre de son principe $»^{4}-$ problème auquel il est bien possible que les sciences sociales - mais peut-on s'en étonner? il s'agit de disciplines qui se veulent scientifiques - n'aient pas de réponse - ou du moins pas d'autre réponse que de décrire des phénomènes qui sont autant de facettes de la dépossession du sens. Nous n'aurions plus alors qu'à tenter de rendre compte des fonctions que nous assignons à ces descriptions.

2. Quelles fonctions? - L'une ou l'autre, les unes et les autres, des trois fonctions suivantes:

- Comprendre - comprendre les phénomènes de dépossession du sens, tenter d'en établir des typologies au delà des différents phénomènes sous lesquels ils se présentent et par lesquels ils se manifestent; comprendre donc:

l' effet d'absurdité qui ne tient plus simplement comme chez Kafka à l'impossibilité pour l'individu de se trouver reconnu par le système, alors que celui-ci lui fournit les repères d'une quête de sens toujours incomplète, mais qui provient des effets systémiques qui, pourtant alimentés par les interactions signifiantes, jouent le rôle de forces de rappel qui vouent les tentatives d'exploration signifiantes à l'échec;

la réduction de l'effet de transcendance à l'effet de dépossession. La Loi, même dans ce rapport absurde, en imposait (de même pour l'Etat, la richesse

4 J. Derrida, Force de loi, le fondement mystique de l'autorité, Cardozo Law School Review, Vol. 11, July-August 1990, n 5-6, p. 960. 
nationale, etc.). Maintenant, elle suit les contraintes internes du système juridique; ou bien, inversement, elle ajoute dispositions sur dispositions dans une course poursuite avec les utilisateurs des failles de la loi;

l'acceptation par les producteurs de significations de leur instrumentalisation, qui n'est que leur insertion dans les effets systémiques. Cela vaut particulièrement de la recherche. Un biais particulier est celui de la sociologie, où les chercheurs se pensent comme des sortes de thérapeutes qui ne diagnostiquent et ne soignent que les symptômes;

la réduction du symbolique global à des processus micro-économiques, et inversement l'incapacité à distinguer dans les interactions locales les repérages locaux, les contraintes systémiques, et les imaginaires globaux.

- Résister - résister à la souffrance de la dépossession: le salarié se voit comme un licencié futur, et le licencié ne peut même pas penser que le travail donnerait un sens social à son activité. Ils savent tous les deux que l'économie progresse par des différences relatives de productivité, donc que la valeur de leur travail tient seulement à ce qu'il est soit moins coûteux que celui du salarié d'un autre pays, soit à ce qu'il bénéficie d'un apprentissage technologique qui n'est pour l'instant disponible que dans tel réseau.

Les résistances à ces dépossessions peuvent être activistes (altermondialisme); elles ne se pensent alors que comme résistances (anti OGM) au lieu de se penser comme projets alternatifs; ou encore, elles tiennent simplement à ce que les humains continuent à faire des choix selon les émotions qu'ils en anticipent, continuent à préférer une dépense agie à une dépense subie, continuent à éprouver des émotions plus fortes et plus positives quand ils pensent transformer quelque situation du monde selon un gradient de valeur que quand ils n'ont que des comportements de consommateurs dont la seule action est de choisir entre les biens qui leur sont proposés.

- Juger - qui est peut-être celle des fonctions - «Devant la loi » en témoigne à sa manière à la fois énigmatique et dramatique - à laquelle les sciences sociales nous préparent le mieux et devant laquelle nous sommes pourtant le plus désarmés.

Certes, on peut considérer que ces effets de dépossession tiennent à des contrastes historiques. Après de très longues périodes où il était considéré comme normal que le sens ne soit pas en notre possession (religion ou platonisme), la période des Lumières et plus encore la révolution industrielle et scientifique ont fait croire que les humains pouvaient disposer de leur sens. Nous ne ferions que sortir de cette illusion. En mathématiques, le passage du réalisme à l'intuitionnisme ou au constructivisme n'a pas eu cette signification, puisqu'en s'imposant des contraintes intuitionnistes, on doit au contraire renoncer à certaines démonstrations. En revanche, les progrès des neurosciences, en approchant des bases neuronales de nos processus de compréhension des significations, nous font osciller entre l'excitation d'expliquer comment nous pouvons saisir du sens, et la dépossession du sens qu'impliquerait l'identification de ces mécanismes et de leurs limites contingentes. La dépossession du sens peut donc être relativisée, au 
regard des mathématiciens et des neurophysiologues. Elle se fait davantage sentir, semble-t-il, dans le domaine des autres sciences sociales, et pourrait sans doute trouver de nouveaux champs d'investigation et de réflexion en particulier du côté des historiens. Et peut-être même des historiens de l'art.

Gérard TIMSIT, Université de Paris 1 Panthéon Sorbonne Pierre LIVET, Université de Provence 